\title{
Similar Connotation in Chronic Hepatitis B and Nonalcoholic Fatty Liver Patients with Dampness-Heat Syndrome
}

\author{
Jianye Dai, ${ }^{1}$ Shujun Sun, ${ }^{1}$ Jianmei Cao, ${ }^{2}$ Yu Zhao, ${ }^{2}$ Huijuan Cao, ${ }^{1}$ Ningning Zheng, \\ Junwei Fang, ${ }^{1}$ Yang Wang, ${ }^{1}$ Wei Zhang, ${ }^{3}$ Yongyu Zhang, ${ }^{1}$ Yiyang $\mathrm{Hu}^{2}{ }^{2}$ and Zhiwei $\mathrm{Cao}^{4}$ \\ ${ }^{1}$ Center for Traditional Chinese Medicine and Systems Biology, Shanghai University of Traditional Chinese Medicine, \\ Shanghai 201203, China \\ ${ }^{2}$ Institute of Liver Diseases, Shuguang Hospital, Key Laboratory of Liver and Kidney Diseases of Ministry of Education, \\ Shanghai University of Traditional Chinese Medicine, Shanghai 201203, China \\ ${ }^{3}$ Liver Department, Longhua Hospital, Shanghai University of Traditional Chinese Medicine, Shanghai 200032, China \\ ${ }^{4}$ School of Life Sciences and Technology, Tongji University, Shanghai 200092, China \\ Correspondence should be addressed to Yongyu Zhang; dryyz@sina.com and Yiyang Hu; yyhuliver@163.com
}

Received 10 January 2013; Revised 2 March 2013; Accepted 4 March 2013

Academic Editor: Aiping Lu

Copyright (C) 2013 Jianye Dai et al. This is an open access article distributed under the Creative Commons Attribution License, which permits unrestricted use, distribution, and reproduction in any medium, provided the original work is properly cited.

The phenomenon that the same syndrome turns up in different diseases appears in the sight of people around the world, which raises the thought for possibility of "Same Treatment for Different Diseases." Actually, treatment based on ZHENG classification in Traditional Chinese Medicine could bring revelation for the former finding. The dampness-heat syndrome in chronic hepatitis B and nonalcoholic fatty liver is regarded as the breakthrough point. We discussed the molecular mechanism of similar connotation that exists in chronic hepatitis B and nonalcoholic fatty liver by metabonomics to give the modern understanding of dampness-heat syndrome. Both urine and serum metabolic profiling revealed that obvious differences existed between dampness-heat syndrome and non-dampness-heat syndrome but the commonality was proved to appear in chronic hepatitis B and nonalcoholic fatty liver patients with dampness-heat syndrome. Furthermore, disorder of body fluid metabolism, decline in digestive capacity, and imbalance of intestinal flora were found to be the new guiding for treatment, with the hope to provide the basis for Chinese personalized medicine.

\section{Introduction}

The phenomenon that different diseases reveal some homogeneity in symptoms $[1,2]$ is raising more and more attention, recently. More importantly, it suggests that different diseases with one or more similar pathological features may be treated with the same drugs $[3,4]$. Actually, traditional Chinese medicine (TCM) participators have found and applied therapeutic principles of "Same Treatment for Different Diseases" for almost hundreds of years, which have been recorded in Treatise on Cold Damage Diseases (Shang Han Lun) and Synopsis of Prescriptions of the Golden Chamber (Jin Gui Yao Lue) $[5,6]$. The same treatment for different diseases means applying the same treatment principles to patients with different kinds of disease only if with the same ZHENG
[7]. What guides treatment is called ZHENG, a temporary state at one time and which is like a syndrome defined by symptoms and signs $[8,9]$. The different diseases in western medicine can manifest in the same ZHENG and vice versa [10].

However, in TCM, one of the most common ZHENGs is dampness-heat syndrome ( $\mathrm{DH})$ which is thought to be caused by a combination of dampness and heat, either of external or of internal origin, with different manifestations according to location, for example, jaundice when dampnessheat accumulates in the liver and gallbladder and diarrhea for dampness-heat in the intestines [7]. Especially, it frequently occurs in Chronic hepatitis B (CHB) and nonalcoholic fatty liver (NFL), with $12.1 \%$ [11] and $37.1 \%$ [12] percentage respectively. 
Since ZHENGs are frequently considered as phenotypes of a disease, researchers are mostly focused on the different ZHENGs in the same disease. However, we should pay more attention to the formerly mentioned phenomenon that the same ZHENG appears in different diseases. This part will bring more inspirations for broadening the application range of some drugs which are suitable for certain ZHENG. In this study, we discussed the molecular mechanisms of the DH in both CHB and NFL by metabonomics. It will be primarily serving for TCM diagnosis and treatment.

\section{Experimental}

2.1. Subjects and Experiment Design. Twenty healthy volunteers and eighty patients of dampness-heat syndrome chronic hepatitis B (DHHB), non-dampness-heat syndrome chronic hepatitis B (NDHHB), dampness-heat syndrome nonalcoholic fatty liver (DHFL), and non-dampness-heat syndrome nonalcoholic fatty liver (NDHFL) were enrolled in the study. The clinical study was approved by the local ethics committee and all of the recruited persons were given informed consent. Diagnostic standards of HB and FL patients were referred to "The guideline of prevention and treatment for chronic hepatitis B" [13] and "Guidelines for management of nonalcoholic fatty liver disease: an updated and revised edition" [14]. Cases meeting the diagnostic criteria for CHB and NFL, respectively, at 18-65 years of age and that would like to sign the informed consent form were included in the study. In addition, there is an exclusion criterion: (1) cases complicated with other hepatotropic virus hepatitis and alcoholic fatty liver, (2) chronic severe hepatitis, (3) $\mathrm{HB}$ and FL patients, associated with serious primary disease of heart, kidney, lung, endocrine, blood, or metabolic and gastrointestinal, or psychotic patients and (4) pregnant or lactating women. A junior medical physician made the initial diagnosis and recorded the information of four traditional examinations accurately and completely. Three more senior physicians (either chief or deputy physicians) subsequently confirmed the initial diagnosis by the records and gave the hierarchical results of typical degree. Only those cases that were identified as classical $\mathrm{DH}$ patients by both the junior and the senior physicians were included in the study to guarantee the correctness of ZHENG differentiation.

2.2. Chemicals and Drugs. N,O-Bis(trimethylsilyl)trifluoroacetamide (BSTFA with $1 \%$ TMCS), urease, L-2-chlorophenylalanine, and heptadecanoic acid were purchased from Sigma-Aldrich Co. LLC (USA). Methoxyamine hydrochloride, methanol, ethanol, myristic acid, chloroform, and pyridine were analytical grade from China National Pharmaceutical Group Corporation (Shanghai, China).

2.3. Sample Collection and Preparation. These patients were in ambulatory care. We collected the outpatients by the recommendation or reservation of doctor. A complete physical examination was given; the health condition was recorded on a scale including the information obtained through
TABLE 1: Temperature program of column incubator in GC/MS.

\begin{tabular}{lccc}
\hline Project & $\begin{array}{c}\text { Rate } \\
\left({ }^{\circ} \mathrm{C} / \mathrm{min}\right)\end{array}$ & $\begin{array}{c}\text { Temperature } \\
\left({ }^{\circ} \mathrm{C}\right)\end{array}$ & $\begin{array}{c}\text { Hold time } \\
(\mathrm{min})\end{array}$ \\
\hline \multirow{3}{*}{ Urine } & & 70 & 2 \\
& 2.5 & 160 & 0 \\
\hline \multirow{4}{*}{ Serum } & 5 & 240 & 16 \\
& 5 & 80 & 2 \\
& 25 & 240 & 0 \\
\hline
\end{tabular}

four traditional examination methods: looking, listening and smelling, asking, and touching at the patient's entry into the study.

And the urina sanguinis and serum were collected from all enrolled subjects. Urine and serum samples were stored at $-80^{\circ} \mathrm{C}$ until GC-MS assay. All these samples were thawed in ice-water bath and vortex-mixed before analysis.

Each $1 \mathrm{~mL}$ aliquot of standard mixture or urine sample was placed into a screw tube, $10 \mathrm{~min}$ centrifugation $(12,000 \mathrm{rpm})$ was given, and $150 \mu \mathrm{L}$ supernatants were transferred into another screw tube. After adding $70 \mu \mathrm{L}$ of urease $(4 \mathrm{mg} / \mathrm{mL})$ and vortex-mixing for $30 \mathrm{~s}$, samples were conditioned at $37^{\circ} \mathrm{C}$ for $15 \mathrm{~min}$ to remove the urea. After adding $800 \mu \mathrm{L}$ of methanol and $10 \mu \mathrm{L}$ of myristic acid in methanol $(1 \mathrm{mg} / \mathrm{mL})$ and mixing for $1 \mathrm{~min}$, the solution was centrifuged at $13,000 \mathrm{rpm}$ for $10 \mathrm{~min}$.

Each $100 \mu \mathrm{L}$ serum sample was transferred into a screw tube. After adding $10 \mu \mathrm{L}$ of L-2-chlorophenylalanine, $10 \mu \mathrm{L}$ of heptadecanoic acid, $300 \mu \mathrm{L}$ of solvent (methanol: chloroform, $3: 1, \mathrm{~V} / \mathrm{V}$ ) and vortex-mixing for $30 \mathrm{~s}$, samples were conditioned at $-20^{\circ} \mathrm{C}$ for $10 \mathrm{~min}$ to precipitate protein. Then the solution was centrifuged at $13,000 \mathrm{rpm}$ for $10 \mathrm{~min}$.

Then a $200 \mu \mathrm{L}$ aliquot of supernatant was transferred into a GC vial and evaporated to dryness under $\mathrm{N}_{2}$ at $30^{\circ} \mathrm{C}$. Methoxyamine in pyridine $(15 \mathrm{mg} / \mathrm{mL})$ was added to the GC vial vortex-mixed for $1 \mathrm{~min}$, and the methoximation reaction was carried out for $90 \mathrm{~min}$ rocking in a shaker at $30^{\circ} \mathrm{C}$; then $50 \mu \mathrm{L}$ of BSTFA plus $1 \%$ TMCS were added to the samples for trimethylsilylation for another $1 \mathrm{~h}$ at $70^{\circ} \mathrm{C}$. At last, the solution was analyzed utilizing GC-MS after vortex for $30 \mathrm{~s}$.

2.4. Data Acquisition. All GC-MS analyses were performed by a mass spectrometer 5975B (Agilent Technologies, USA) coupled to an Agilent 6890 (Agilent Technologies, USA) gas chromatography instrument. In the gas chromatographic system, a capillary column (Agilent J\&W DB-5 ms Ultra Inert $30 \mathrm{~m} \times 0.25 \mathrm{~mm}$, film thickness $0.25 \mu \mathrm{m}$ ) was used. Helium carrier gas was used at a constant flow rate of $1.0 \mathrm{~mL} \times$ $\min ^{-1}$. One $\mu \mathrm{L}$ of derivatized samples was injected into the GC/MS instrument, and splitless injection mode was used. A programmed column temperature was optimized to acquire a well separation, which was demonstrated in Table 1 . The temperatures of the injection port, the interface, and source temperature were set at $280^{\circ} \mathrm{C}, 260^{\circ} \mathrm{C}$, and $230^{\circ} \mathrm{C}$, respectively. The measurements were made with electron 
TABLE 2: The list of subjects' features and liver function indicators.

\begin{tabular}{|c|c|c|c|c|c|}
\hline Indexes & Control & DHHB & NDHHB & DHFL & NDHFL \\
\hline Gender (female/male) & $3 / 17$ & $4 / 16$ & $3 / 17$ & $2 / 18$ & $6 / 14$ \\
\hline Age (year) & $39.20 \pm 13.64$ & $42.35 \pm 15.23$ & $43.05 \pm 11.65$ & $38.30 \pm 17.24$ & $42.15 \pm 12.26$ \\
\hline Stature $(\mathrm{cm})$ & $169.10 \pm 7.77$ & $170.10 \pm 5.60$ & $167.45 \pm 5.39$ & $171.9 \pm 5.67$ & $166.00 \pm 8.55$ \\
\hline Weight (kg) & $61.15 \pm 7.29$ & $64.35 \pm 10.68$ & $66.40 \pm 13.69$ & $68.55 \pm 7.93$ & $61.70 \pm 11.25$ \\
\hline Alanine transaminase $(\mathrm{U} / \mathrm{L})$ & $19.05 \pm 5.25$ & $69.30 \pm 85.96$ & $66.30 \pm 65.57$ & $43.70 \pm 25.39$ & $48.55 \pm 32.56$ \\
\hline Aspartate aminotransferase (U/L) & $19.47 \pm 4.01$ & $52.50 \pm 40.32$ & $51.25 \pm 44.92$ & $29.40 \pm 12.14$ & $39.50 \pm 24.81$ \\
\hline Gamma glutamyltransferase (U/L) & $18.53 \pm 4.48$ & $40.70 \pm 26.04$ & $85.75 \pm 142.10$ & $57.05 \pm 50.66$ & $32.20 \pm 20.51$ \\
\hline Alkaline phosphatase (U/L) & $61.53 \pm 15.47$ & $96.20 \pm 37.40$ & $84.70 \pm 24.97$ & $66.30 \pm 15.90$ & $79.00 \pm 17.32$ \\
\hline
\end{tabular}

TABLE 3: The compound number list of differential urinary metabolites in four ZHENGs.

\begin{tabular}{lc}
\hline Group & Compound number \\
\hline U-DHHB & 20 \\
U-NDHHB & 8 \\
U-DHFL & 23 \\
U-NDHFL & 12 \\
\hline
\end{tabular}

impact ionization $(70 \mathrm{eV})$ in the full scan mode $(\mathrm{m} / \mathrm{z} 30$ 550). The solvent delay time was set to $5 \mathrm{~min}$. The GC-MS operating condition was the same as the previous experiment [15] except for the column temperature program.

2.5. Data Analysis. Due to experimental variations and column aging, shifts in retention time between fingerprints occurred. When the total ion current chromatograms (TICs) were obtained, peak-alignment or warping techniques were commonly applied to compensate for minor shifts in retention times. Thus, in the subsequently data processing, the same variable manifested synchronous information in every profile. So all the GC-MS raw files after being converted to CDF format via the software, which came with Agilent MSD workstation, were subsequently processed by the XCMS toolbox (http://metlin.scripps.edu/download/) using XCMS's default settings with the following exceptions: xcmsSet (full width at half-maximum: fwhm $=5 ; \mathrm{S} / \mathrm{N}$ cutoff value: snthresh $=10, \max =25)$, group $(b w=5)$. The resulting table (CSV file) was exported into Microsoft Excel (Microsoft Inc., USA), where normalization was performed prior to multivariate analyses. The resulting three-dimensional matrix involving peak index (RT-m/z pair), sample names (observations), and normalized peak area percent was introduced into Simca-P 11.5 Software (Umetrics, Umea, Sweden) for the analysis of principal component analysis (PCA), partial least squares discriminant analysis (PLS-DA), and orthogonal partial least squares (OPLS). Differential variables with VIP values [16] exceeding 1.5 between two different groups could be generated from OPLS loadings plot. Subsequently, those variables were further analyzed by Mann-Whitney $U$ test to confirm the changed metabolites by SPSS 17.0 (SPSS, Chicago, IL, USA) with the threshold of $P$ value set at 0.1 . Those variables, then, were identified by searching in NIST 2005 database and verified by standards. The Kyoto Encyclopedia of Genes and Genomes (KEGG) (http://www.genome.ad.jp/kegg/) and Human Metabolome Database (HMDB) (http://www.hmdb.ca/) were used to give the biochemical interpretation of changed metabolites. Then, further analysis was performed by Metabolites Biological Role (MBRole) (http://csbg.cnb.csic.es/mbrole/).

\section{Results}

3.1. Clinical Information. The clinical information of all five groups was showed in Table 2, such as subjects' features and liver function indicators. There was no significant difference between every two syndromes in each disease.

3.2. Difference Analysis of Urinary Metabolic Profiles. Figure 1 depicted score plot of orthogonal partial least squares (OPLS) of urine. It could be found that DHHB, NDHHB, DHFL, and NDHFL groups were clearly separated from the control group. The most meaningful characteristics were screened by orthogonal partial least squares (OPLS) loading plot analysis, which could effectively extract variables responsible for the separation by removing variables unrelated to pathological status. The quality of the model was characterized by two performance statistics, $R^{2} Y$ (cum) and $Q^{2} Y$ (cum), indicating the total explanation and predictability of the model [17]. The information of models was summarized in Table 7. Due to space limitation, the detailed information of selected urinary differential metabolites was summarized in Supplementary Information (SI) available at http://dx.doi.org/10.1155/2013/793820. But the brief information was listed in Table 3. Furthermore, the related pathways of each group were analyzed by KEGG and MBRole as followed, based on the selected differential metabolites (Table 4).

3.3. Difference Analysis of Serum Metabolic Profiles. The score plot of OPLS of serum was depicted in Figure 2 where it could be found that DHHB, NDHHB, DHFL, and NDHFL groups were clearly separated from the control group. Then the most meaningful characteristics of every group were screened out by OPLS loading analysis. The information 
TABLE 4: The information of changed pathways in urine of four ZHENGs.

\begin{tabular}{|c|c|c|c|}
\hline Label & Group & Class $^{\mathrm{a}}$ & $P$ value $^{\mathrm{b}}$ \\
\hline Butanoate metabolism & U-DHHB & Carbohydrate metabolism & 0.01 \\
\hline Starch and sucrose metabolism & U-DHHB & Carbohydrate metabolism & 0.01 \\
\hline Pentose and glucuronate interconversions & U-DHHB & Carbohydrate metabolism & 0.01 \\
\hline $\mathrm{ABC}$ transporters & U-DHHB & Signal transduction & 0.00 \\
\hline Pentose and glucuronate interconversions & U-NDHHB & Carbohydrate metabolism & 0.00 \\
\hline $\mathrm{ABC}$ transporters & U-NDHHB & Signal transduction & 0.01 \\
\hline Phenylalanine metabolism & U-DHFL & Amino acid metabolism & 0.03 \\
\hline Butanoate metabolism & U-DHFL & Carbohydrate metabolism & 0.02 \\
\hline Galactose metabolism & U-DHFL & Carbohydrate metabolism & 0.02 \\
\hline $\mathrm{ABC}$ transporters & U-DHFL & Signal transduction & 0.09 \\
\hline Benzoate degradation & U-DHFL & Xenobiotics biodegradation and metabolism & 0.04 \\
\hline Amino sugar and nucleotide sugar metabolism & U-NDHFL & Carbohydrate metabolism & 0.01 \\
\hline Pentose and glucuronate interconversions & U-NDHFL & Carbohydrate metabolism & 0.00 \\
\hline
\end{tabular}

${ }^{a}$ The classes are categorized by KEGG.

${ }^{\mathrm{b}} P$ value is obtained from analysis of MBRole.

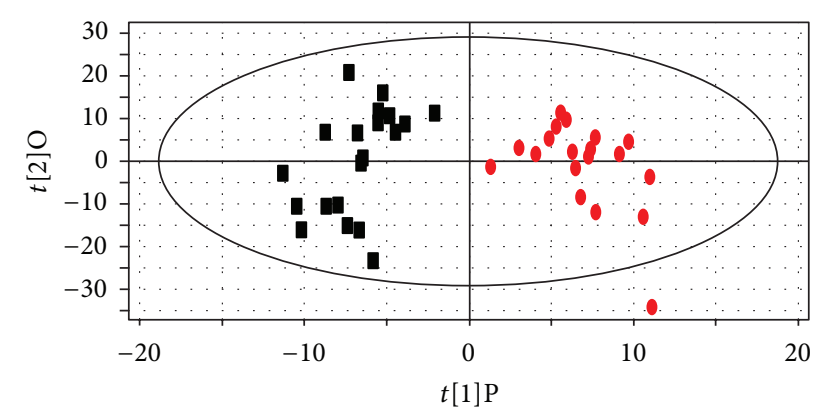

$R^{2} X[1]=0.0881377 \quad R^{2} X[2]=0.305159 \quad$ Ellipse: hotelling

- Control

- DHHB

(a)

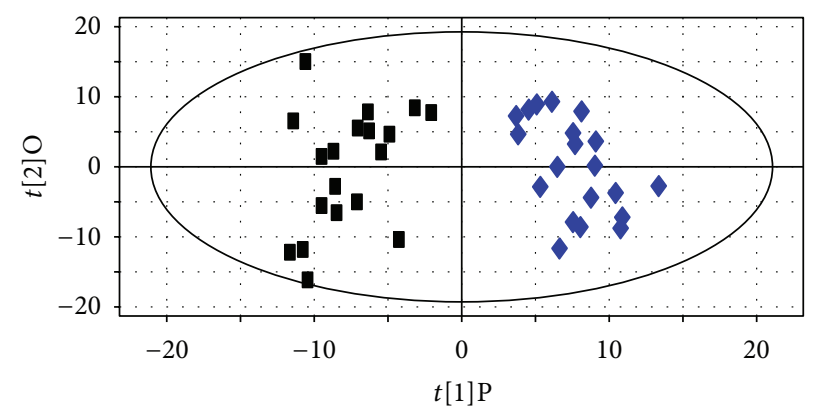

$R^{2} X[1]=0.109919 \quad R^{2} X[2]=0.252084 \quad$ Ellipse: hotelling T2 (0.95)

- Control

$\checkmark$ DHFL

(c)

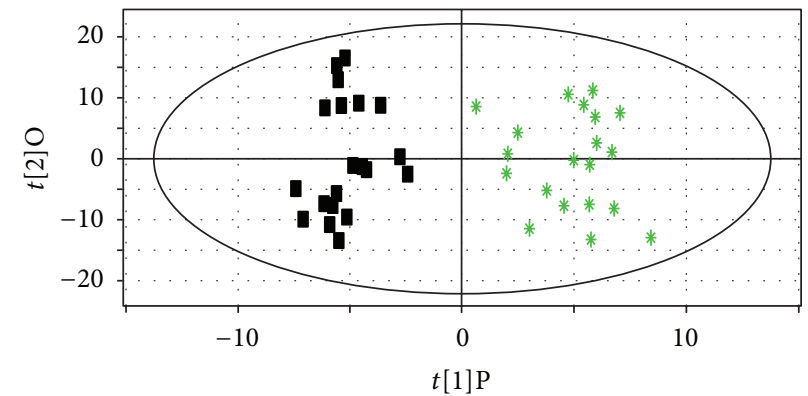

$R^{2} X[1]=0.0473386 R^{2} X[2]=0.187676 \quad$ Ellipse: hotelling T2 (0.95)

- Control

* NDHHB

(b)



$R^{2} X[1]=0.0722322 \quad R^{2} X[2]=0.267058 \quad$ Ellipse: hotelling T2 (0.95)

- Control

$\triangle$ NDHFL

(d)

FIGURE 1: OPLS score plot of four ZHENGs compared to healthy control group by urinary metabolic profiles. (a) OPLS score plot between control and DHHB. (b) OPLS score plot between control and NDHHB. (c) OPLS score plot between control and DHFL. (d) OPLS score plot between control and NDHFL. 


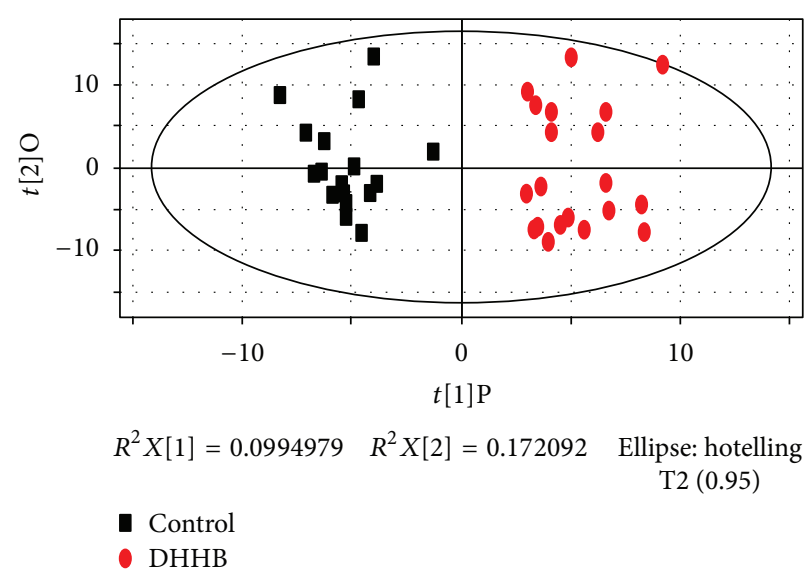

(a)



(c)

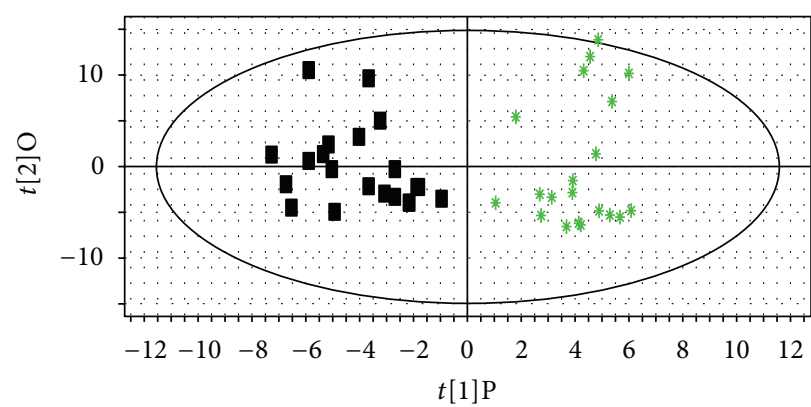

$R^{2} X[1]=0.068731 \quad R^{2} X[2]=0.155264 \quad$ Ellipse: hotelling T2 (0.95)

- Control

* NDHHB

(b)

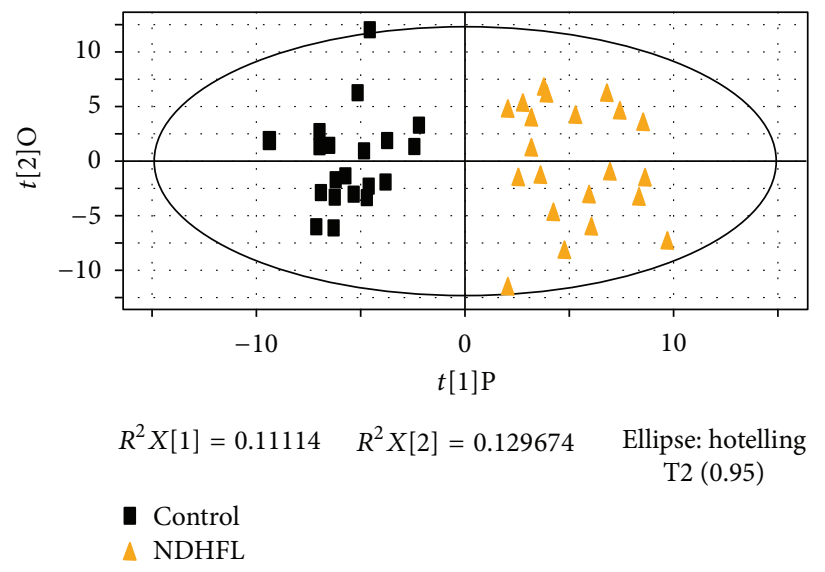

(d)

FIGURE 2: OPLS score plot of four ZHENGs compared to healthy control group by serum metabolic profiles. (a) OPLS score plot between control and DHHB. (b) OPLS score plot between control and NDHHB. (c) OPLS score plot between control and DHFL. (d) OPLS score plot between control and NDHFL.

TABLE 5: The compound number list of differential serum metabolites in four ZHENGs.

\begin{tabular}{lc}
\hline Group & Compound number \\
\hline S-DHHB & 33 \\
S-NDHHB & 20 \\
S-DHFL & 26 \\
S-NDHFL & 23 \\
\hline
\end{tabular}

of models was summarized in Table 7. The detailed information of serum differential metabolites was also summarized in SI, and the brief information was listed in Table 5. Furthermore, the corresponding pathways of each group were analyzed by KEGG and MBRole as follows, based on the selected differential metabolites (Table 6). The partial least squares discriminant analysis (PLS-DA) score plot of either urine or serum profiling could reveal that DHHB and DHFL clustered in a region (circled in Figure 3) and straggled with the other NDH groups. It might suggest that DHHB and DHFL have more commonalities which may be the connotation of DH. Given that all groups of selected markers may contain information of ZHENG and disease, the biomarkers of DH should be filtered further. Based on the thought of the "Same Disease with Different ZHENGs," the potential biomarkers of a ZHENG in one disease could be selected by excluding the intersection of two or more ZHENGs of this disease. Therefore, $\mathrm{DH}$ potential biomarkers in $\mathrm{CHB}$ and NFL were selected, respectively, with the aforementioned process. Then we took advantage of the "Different Diseases with Same ZHENG," to select the intersection of two upper groups of biomarkers as the final $\mathrm{DH}$ potential biomarkers (Table 8 ). The schematic diagram was shown by Figure 4. And the unique pathways of $\mathrm{DH}$, which were obtained by the same method as above, were listed in Table 9. The respectively obtained urinary and serum information is integrated to interpret the connotation of $\mathrm{DH}$ 
TABLE 6: The information of changed pathways in serum of four ZHNEGs.

\begin{tabular}{|c|c|c|c|}
\hline Label & Group & Class $^{\mathrm{a}}$ & $P$ value $^{\mathrm{b}}$ \\
\hline Alanine, aspartate, and glutamate metabolism & S-DHHB & Amino acid metabolism & 0.02 \\
\hline Arginine and proline metabolism & S-DHHB & Amino acid metabolism & 0.00 \\
\hline Glycine, serine, and threonine metabolism & S-DHHB & Amino acid metabolism & 0.01 \\
\hline Lysine biosynthesis & S-DHHB & Amino acid metabolism & 0.03 \\
\hline Lysine degradation & S-DHHB & Amino acid metabolism & 0.00 \\
\hline Phenylalanine, tyrosine, and tryptophan biosynthesis & S-DHHB & Amino acid metabolism & 0.02 \\
\hline Novobiocin biosynthesis & S-DHHB & Biosynthesis of other secondary metabolites & 0.03 \\
\hline Streptomycin biosynthesis & S-DHHB & Biosynthesis of other secondary metabolites & 0.02 \\
\hline Galactose metabolism & S-DHHB & Carbohydrate metabolism & 0.00 \\
\hline Nitrogen metabolism & S-DHHB & Energy metabolism & 0.00 \\
\hline Biosynthesis of unsaturated fatty acids & S-DHHB & Lipid metabolism & 0.00 \\
\hline Fatty acid biosynthesis & S-DHHB & Lipid metabolism & 0.06 \\
\hline Thiamine metabolism & S-DHHB & Metabolism of cofactors and vitamins & 0.02 \\
\hline Cyanoamino acid metabolism & S-DHHB & Metabolism of other amino acids & 0.00 \\
\hline Glutathione metabolism & S-DHHB & Metabolism of other amino acids & 0.00 \\
\hline Pyrimidine metabolism & S-DHHB & Nucleotide metabolism & 0.08 \\
\hline $\mathrm{ABC}$ transporters & S-DHHB & Signal transduction & 0.00 \\
\hline Aminoacyl-tRNA biosynthesis & S-DHHB & Signal transduction & 0.00 \\
\hline Two-component system & S-DHHB & Signal transduction & 0.02 \\
\hline $\mathrm{ABC}$ transporters & S-DHHB & Signal transduction & 0.01 \\
\hline Arginine and proline metabolism & S-NDHHB & Amino acid metabolism & 0.00 \\
\hline Glycine, serine, and threonine metabolism & S-NDHHB & Amino acid metabolism & 0.03 \\
\hline Lysine degradation & S-NDHHB & Amino acid metabolism & 0.03 \\
\hline Galactose metabolism & S-NDHHB & Carbohydrate metabolism & 0.02 \\
\hline Pentose and glucuronate interconversions & S-NDHHB & Carbohydrate metabolism & 0.00 \\
\hline Biosynthesis of unsaturated fatty acids & S-NDHHB & Lipid metabolism & 0.00 \\
\hline Fatty acid biosynthesis & S-NDHHB & Lipid metabolism & 0.03 \\
\hline Nicotinate and nicotinamide metabolism & S-NDHHB & Metabolism of cofactors and vitamins & 0.03 \\
\hline Glutathione metabolism & S-NDHHB & Metabolism of other amino acids & 0.00 \\
\hline $\mathrm{ABC}$ transporters & S-NDHHB & Signal transduction & 0.00 \\
\hline Aminoacyl-tRNA biosynthesis & S-NDHHB & Signal transduction & 0.01 \\
\hline Arginine and proline metabolism & S-DHFL & Amino acid metabolism & 0.06 \\
\hline Lysine degradation & S-DHFL & Amino acid metabolism & 0.02 \\
\hline Glycolysis/Gluconeogenesis & S-DHFL & Carbohydrate metabolism & 0.01 \\
\hline Nitrogen metabolism & S-DHFL & Energy metabolism & 0.01 \\
\hline Thiamine metabolism & S-DHFL & Metabolism of cofactors and vitamins & 0.01 \\
\hline Cyanoamino acid metabolism & S-DHFL & Metabolism of other amino acids & 0.00 \\
\hline Glutathione metabolism & S-DHFL & Metabolism of other amino acids & 0.01 \\
\hline Purine metabolism & S-DHFL & Nucleotide metabolism & 0.01 \\
\hline $\mathrm{ABC}$ transporters & S-DHFL & Signal transduction & 0.00 \\
\hline Aminoacyl-tRNA biosynthesis & S-DHFL & Signal transduction & 0.00 \\
\hline Biosynthesis of unsaturated fatty acids & S-NDHFL & Lipid metabolism & 0.00 \\
\hline Fatty acid biosynthesis & S-NDHFL & Lipid metabolism & 0.00 \\
\hline $\mathrm{ABC}$ transporters & S-NDHFL & Signal transduction & 0.01 \\
\hline Aminoacyl-tRNA biosynthesis & S-NDHFL & Signal transduction & 0.01 \\
\hline
\end{tabular}

${ }^{\mathrm{a}}$ The classes are categorized by KEGG.

${ }^{\mathrm{b}} P$ value is obtained from analysis of MBRole. 


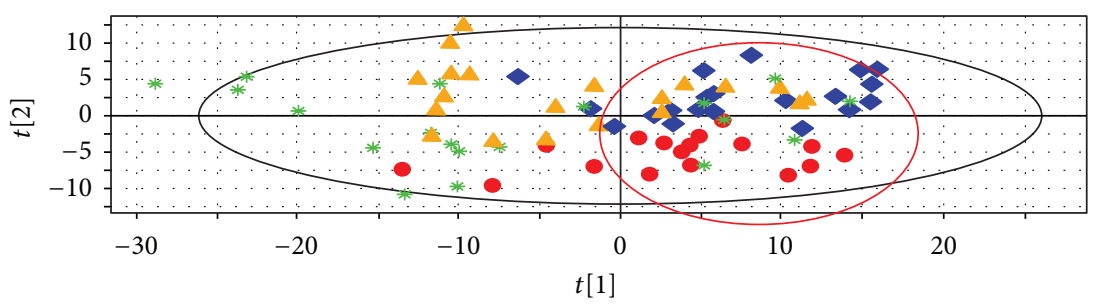

$$
\begin{array}{ll}
R^{2} X[1]=0.204643 & R^{2} X[2]=0.0810892 \quad \text { Ellipse: hotelling T2 (0.95) } \\
\begin{array}{ll}
- \text { DHHB } & * \text { NDHHB }
\end{array} \\
\qquad \text { DHFL } & \triangle \text { NDHFL }
\end{array}
$$

(a)

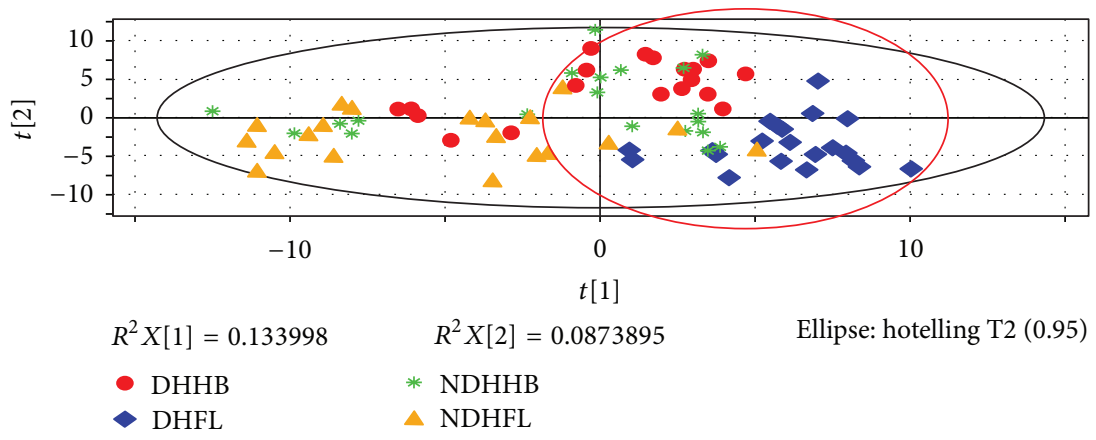

(b)

FIGURe 3: PLS-DA score plot of four ZHENGs. (a) PLS-DA score plot of urinary profiling. (b) PLS-DA score plot of serum profiling.

TABLE 7: Summary of the modeling quality of OPLS analysis.

\begin{tabular}{lccccc}
\hline Name & Group & $\mathrm{No}^{\mathrm{a}}$ & $R^{2} X_{\text {cum }}{ }^{\mathrm{b}}$ & $R^{2} Y_{\text {cum }}{ }^{\mathrm{c}}$ & $\mathrm{Q}^{2} Y_{\text {cum }}{ }^{\mathrm{d}}$ \\
\hline 1A & U-DHHB & $1 \mathrm{P}+2 \mathrm{O}^{\mathrm{e}}$ & 0.50 & 0.89 & 0.70 \\
1B & U-NDHHB & $1 \mathrm{P}+3 \mathrm{O}$ & 0.56 & 0.90 & 0.48 \\
1C & U-DHFL & $1 \mathrm{P}+3 \mathrm{O}$ & 0.49 & 0.91 & 0.57 \\
1D & U-NDHFL & $1 \mathrm{P}+2 \mathrm{O}$ & 0.42 & 0.85 & 0.51 \\
2A & S-DHHB & $1 \mathrm{P}+2 \mathrm{O}$ & 0.36 & 0.91 & 0.67 \\
2B & S-UDHHB & $1 \mathrm{P}+2 \mathrm{O}$ & 0.31 & 0.88 & 0.58 \\
2C & S-DHFL & $1 \mathrm{P}+2 \mathrm{O}$ & 0.31 & 0.91 & 0.52 \\
2D & S-NDHFL & $1 \mathrm{P}+2 \mathrm{O}$ & 0.33 & 0.87 & 0.41 \\
\hline
\end{tabular}

${ }^{a}$ No represents the number of components.

${ }^{b, c} R^{2} X_{\text {cum }}$ and $R^{2} Y_{\text {cum }}$ represent the cumulative sum of squares (SS) of all the $X$ 's and $Y$ 's explained by all extracted components.

${ }^{\mathrm{d}} \mathrm{Q}^{2} Y_{\text {cum }}$ is an estimate of how well the model predicts the $Y$ 's.

${ }^{\mathrm{e}} 1 \mathrm{P}+2 \mathrm{O}$ : one predictive component and two orthogonal components for establishing the OPLS model.

exists in chronic hepatitis B and non-alcoholic fatty liver from the molecular level.

\section{Discussion}

The datasets from serum and urine were combined to get a global view of coanalysis. All the detected potential biomarkers, to a large extent, were caused by deregulated systemic changes and development or progression of $\mathrm{DH}$. After analysis of the unique metabolites of $\mathrm{DH}$, it was revealed that two most types of substances were carboxylic acid and alpha-amino acid. Eight biomarkers (acetic acid, succinic acid, and so on) were classified as carboxylic acid and four (glycine, L-lysine, L-asparagine, and aminolevulinic acid) as alpha-amino acid. Among these biomarkers of $\mathrm{DH}$, only three metabolites (acetic acid, amino levulinic acid, and butyrate) were detected both in the serum and urine. Meanwhile, almost all biomarkers were uniquely serum-derived or urine-derived. This suggested the necessity to combine both serum and urine samples.

The characteristic metabolites of $\mathrm{DH}$ were hoped to be developed as the biomarkers. To achieve this goal, more cases are needed and another disease located in other organs should be incorporated to eliminate the impact of liver disease. After further validation, the filtered biomarkers could provide revelation to the standardization of $\mathrm{DH}$ classification and the subtype's distinction of CHB and NFL. But it is insufficient to explain the connotation of $\mathrm{DH}$ by only these characteristic metabolites. So we should expand our horizons to all the DH-related metabolites and pathways in our study.

After coanalysis of serum and urinary information by KEGG and literature, the schematic diagram of the specific pathways in $\mathrm{DH}$ had been demonstrated in Figure 5. As shown, thiamine metabolism, nitrogen metabolism, cyanoamino acid metabolism, and butanoate metabolism were all downregulated. Furthermore, the statistics were 
TABLE 8: The list of unique metabolites of DH.

\begin{tabular}{lccc}
\hline Compound name & $\mathrm{VIP}^{\mathrm{a}}$ & $P^{\mathrm{b}}$ & $\mathrm{FN}^{\mathrm{c}}$ \\
\hline beta-Aminoisobutyric acid & 1.47 & 0.02 & -1.52 \\
beta-D-Glucopyranose & 1.81 & 0.00 & -1.79 \\
1-Naphthol & 1.79 & 0.01 & -1.64 \\
Butanedioic acid & 2.00 & 0.00 & -1.76 \\
D-Glucose & 1.17 & 0.02 & +1.24 \\
Glycine & 1.86 & 0.00 & -1.87 \\
L-Asparagine & 1.62 & 0.01 & -1.60 \\
L-Lysine & 1.68 & 0.02 & -1.68 \\
Urea & 1.84 & 0.01 & +1.80 \\
1H-Indole-3-acetic acid & 1.65 & 0.00 & -1.91 \\
1H-Indole-3-butanoic acid & 1.65 & 0.01 & -1.59 \\
(R)-Mandelic acid & 1.72 & 0.01 & -1.67 \\
Acetic acid & 1.70 & 0.00 & -1.89 \\
Butyrate & 1.71 & 0.00 & -1.69 \\
Creatinine & 1.71 & 0.01 & -1.66 \\
1-Cyclohexenecarboxylic acid & 1.90 & 0.00 & -1.74 \\
Aminolevulinic acid & 1.61 & 0.00 & -1.79 \\
Glutaconic acid & 1.73 & 0.01 & -1.63 \\
Pteridine & 1.66 & 0.00 & -1.72 \\
Pyrazine & 1.58 & 0.00 & -2.10 \\
Succinic acid & 1.88 & 0.01 & -1.71 \\
\hline VIP:varab & & &
\end{tabular}

${ }^{a}$ VIP: variable importance in the project.

${ }^{\mathrm{b}} P$ value was obtained from Mann-Whitney test (ZHENGs compared to healthy control).

${ }^{\mathrm{c}} \mathrm{FN}$ is fold change of mean ranks calculated by the Mann-Whitney test (ZHENGs compared to healthy control). "+" means upregulated, "-" means downregulated.

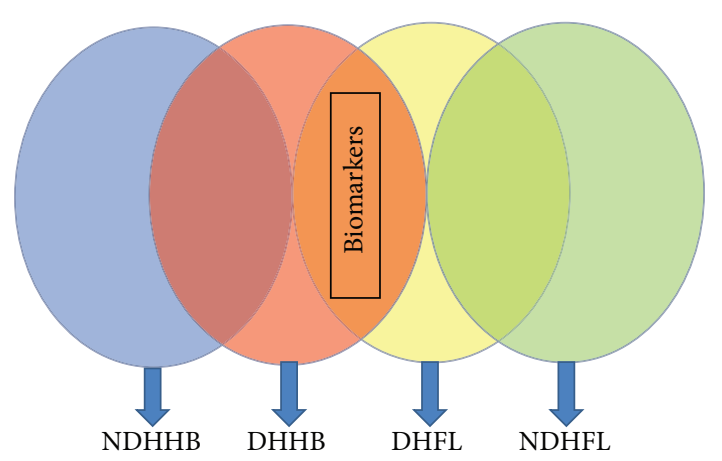

FIGURE 4: Schematic diagram of research approach for selection of $\mathrm{DH}$.

performed for the metabolic modules of urinary and serum pathways in $\mathrm{DH}$ and $\mathrm{NDH}$, as shown in Figure 6. We found that there was an obvious difference between $\mathrm{DH}$ and NDH in patients' metabolic levels. Especially, Biosynthesis of other secondary metabolites, energy metabolism, nucleotide metabolism, and xenobiotics biodegradation and metabolism were the unique metabolic changes. These may demonstrate the molecular mechanism of DH.
In our study, energy metabolism had been found, to have obvious changes, which was revealed by the down-regulated pathways of nitrogen metabolism and butanoate metabolism. As we all know is, energy metabolism ultimately linked to adenosine triphosphate (ATP). Downregulated Acetic acid and upregulated propanoic acid suggest that acetate kinase has been inhibited, which is closely related to ATP [18, 19]. Therefore the down-regulated pathway could be related to ATP insufficiency or decreased ATP enzyme activity, which could lead to a decrease in the $\mathrm{Na}^{+}-\mathrm{K}^{+}$-ATP enzyme activity. These all will result in sodium and potassium content disorders within cells and tissues. Furthermore, it causes liquid water imbalance, tissue edema, and other structural changes, to result in further energy supply shortage [20]. Some researchers have found a the decline of $\mathrm{Na}^{+}-\mathrm{K}^{+}$-ATP enzyme activity in $\mathrm{DH}$ patients' liver mitochondria and erythrocyte membrane [21, 22]. And Aquaporin-2, which is one of the water permeability transporter protein [23], has been reported to be reduced in DH [24]. Above all, body fluid metabolism disorder could be resulted by the accumulation of sodium elements in intracellular fluid, which sounds similar to the "water-dampness stagnation," one of the incentives of the DH in TCM [25].

In addition, we found that carbohydrate digestion and absorption (D-glucose, acetate, butanoic acid), protein digestion and absorption (acetate, glycine, L-lysine, L-asparagine, butanoic acid), and mineral absorption (Dglucose, glycine, L-asparagine) were downregulated, which could be explained as decline in digestive capacity. In addition, the depressed microbial-related pathway, biosynthesis of other secondary metabolites [26], and xenobiotics biodegradation and metabolism $[27,28]$ would reveal the disorder in gastrointestinal bacterial flora, which may be the incentive of functional disturbance in digestion and absorption. In a word, the depressed digestive capacity and intestinal flora imbalance are both thought to be the main reason of "spleenstomach disharmony," another incentive of the DH $[5,6]$. The schematic diagram of molecular mechanism of $\mathrm{DH}$ is demonstrated in Figure 7.

Edema and refractory are two common features of $\mathrm{DH}$, which might be explained by the present results. Thiamine is one of eight members of the B family of vitamins, which is essential to the body to process carbohydrates. As an increase of dietary carbohydrate intake caused a decrease of plasma and urine levels of thiamine without affecting enzyme activities [29], the DH patients, who surfeit sweet and greasy food, will suffer more severe thiamine deficiency. This phenomenon has been proved by our results as down-regulated thiamine metabolism. However, thiamine deficiency would cause joint swelling $[30,31]$. And the accumulation of sodium elements in intracellular fluid as former will make the edema more serious. To refractory, it is worth mentioning that down-regulated xenobiotics biodegradation and metabolism reveals the depression of scavenging endotoxin ability. A study showed that could be caused by inhibition bactericidal/permeability Increasing protein (BPI) mRNA expressing in moisture and heat environment [32], which is external factors of DH. Furthermore, in the vitality of the same bacteria or endotoxin activity conditions, the decrease in 
TABLE 9: The list of unique pathways of DH in CHB and NFL.

\begin{tabular}{|c|c|c|c|c|c|c|}
\hline Label & Sample & $P$ value & \multicolumn{4}{|c|}{ Compound } \\
\hline Cyanoamino acid metabolism & Serum & 0.00 & L-Tyrosine $\downarrow$ & Glycine $\downarrow$ & L-Asparagine $\downarrow$ & L-Aspartic acid $\downarrow$ \\
\hline Nitrogen metabolism & Serum & 0.01 & & Glycine $\downarrow$ & L-Asparagine $\downarrow$ & L-Aspartic acid $\downarrow$ \\
\hline Thiamine metabolism & Serum & 0.02 & L-Tyrosine $\downarrow$ & Glycine $\downarrow$ & & \\
\hline Butanoate metabolism & Urine & 0.01 & & Butyrate $\downarrow$ & Succinic acid $\downarrow$ & \\
\hline
\end{tabular}

The levels of differential metabolites were labeled with $(\downarrow)$ downregulated and $(\uparrow)$ upregulated.

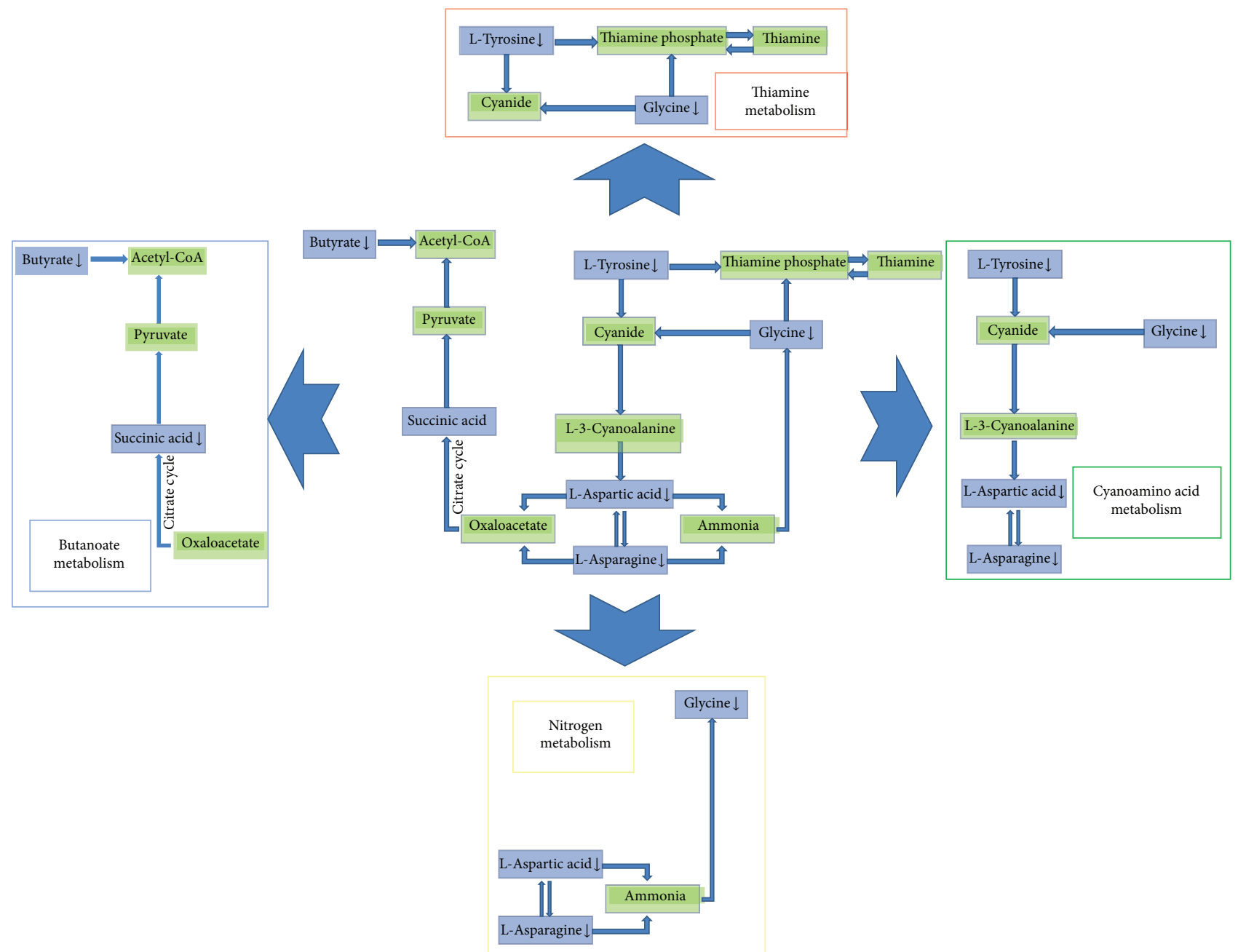

FIGURE 5: The schematic diagram of the unique pathways in DH.

the synthesis and secretion of BPI could induce that the illness may develop or recovery is not easy to be cured.

Based on the former results, we could give our advice to develop new treatment for CHB and NFL. Firstly, the patients should be treated by considering the connotation of $\mathrm{DH}$, when they appear the typical symptoms of $\mathrm{DH}$ such as red tongue and yellow greasy tongue coating. Secondly, the balance of sodium in intracellular fluid could be the new target for DH patients of CHB and NFL. Finally, regulating the function of spleen and stomach and balancing the intestinal flora would be the revelation for the treatment of $\mathrm{CHB}$ and NFL. Therefore, understanding the molecular mechanisms of
$\mathrm{DH}$ in different diseases will be primarily serving for TCM diagnosis and treatment. This research firstly provides the evidence which needs to be proved by further research with more samples.

\section{Conclusion}

In this study, we conducted urine and serum metabonomics to explore the molecular mechanism of dampness-heat syndrome in CHB and NFL. After screening out the differential urine and serum metabolites of DHHB, NDHHB, DHFL, 


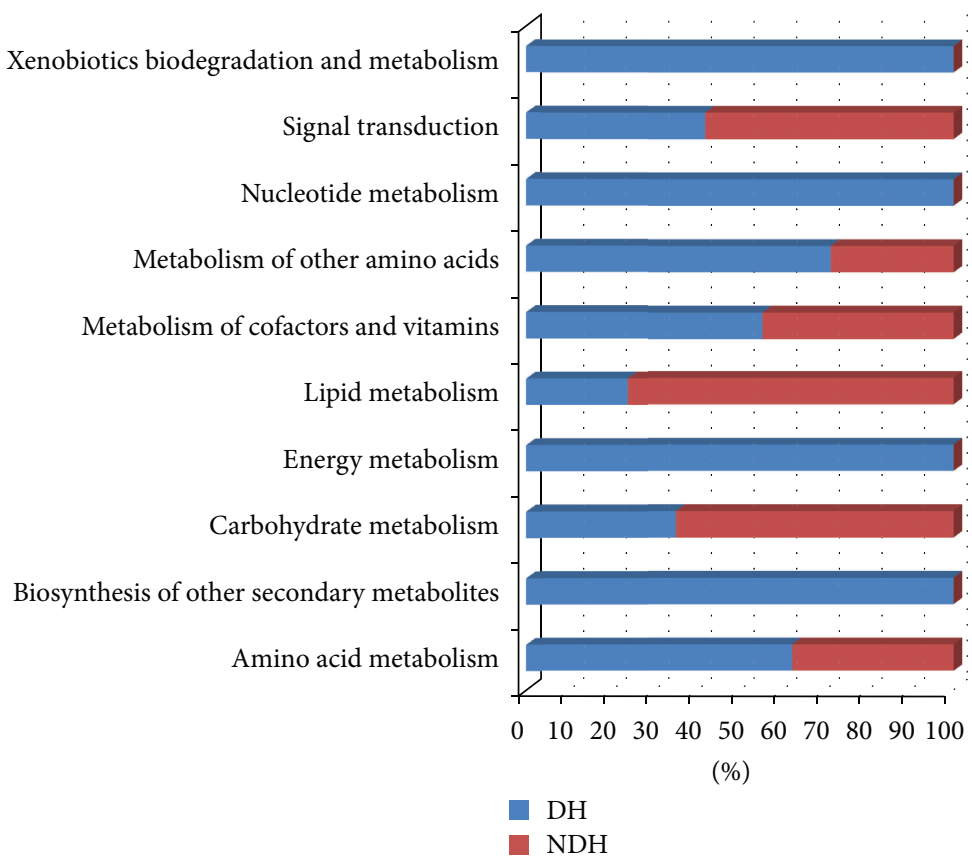

FIGURE 6: Comparison charts of metabolic functional modules between DH and NDH.

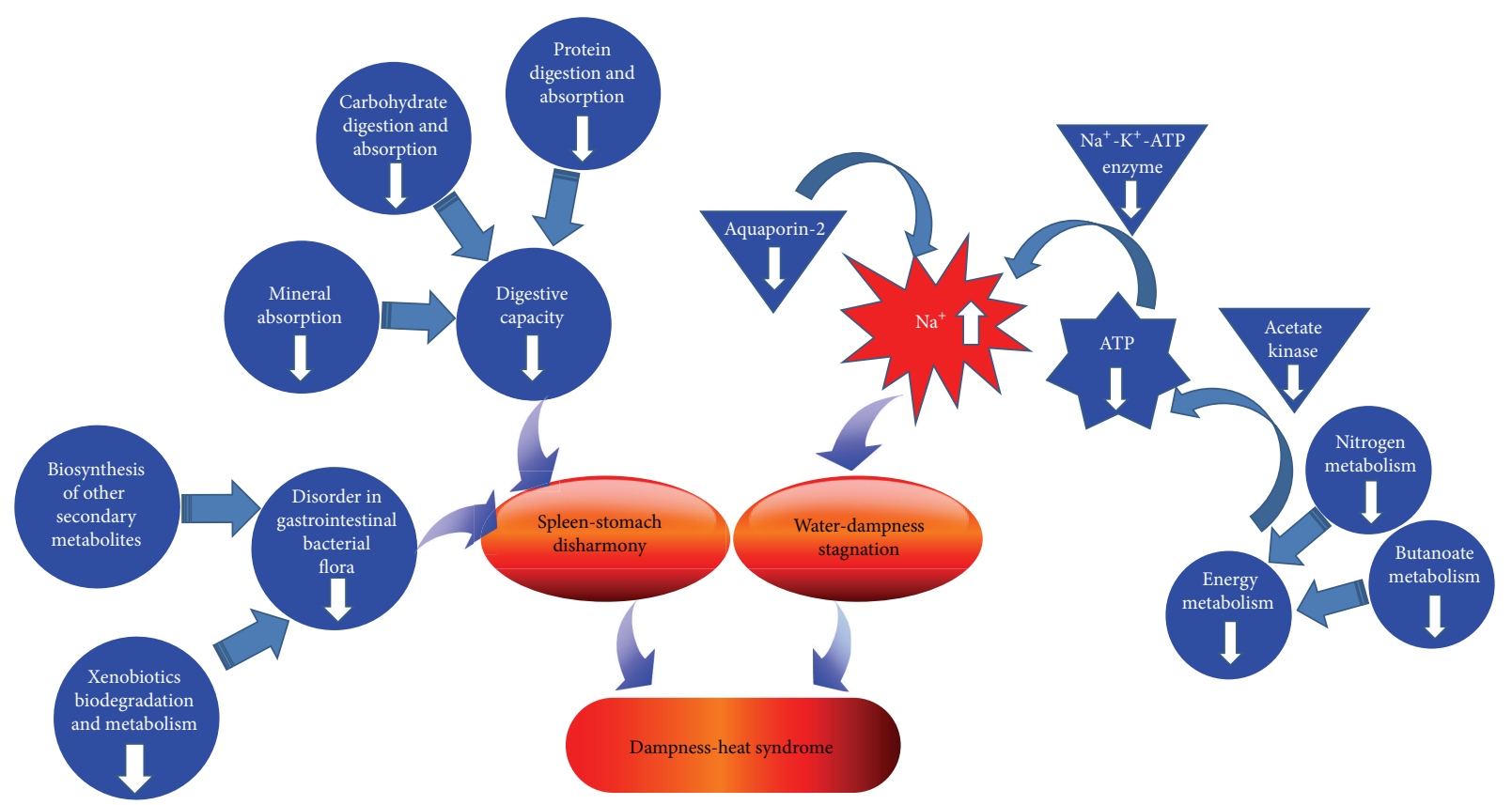

FIgURE 7: The schematic diagram of molecular mechanism of DH.

and NDHFL, the pathways of each group were analyzed. There were obvious differences found between $\mathrm{DH}$ and $\mathrm{NDH}$ in the metabolic level. And the commonality was proved to exist in CHB and NFL patients with DH. By two steps, the disease information was removed to reserve the characteristics of DH. Further studies showed that body fluid metabolism disorder resulted by the accumulation of sodium elements in intracellular fluid, depressed digestive capacity, and intestinal flora imbalance could be the main reasons for
$\mathrm{DH}$. We hope that the results could provide new revelation for treatment of $\mathrm{CHB}$ and NFL and a basis for expanding application of old drugs.

\section{Authors' Contribution}

J. Dai, S. Sun, J. Cao, and Y. Zhao contributed equally to this work and should be considered as co-first authors. 


\section{Conflict of Interests}

The authors declared that they have no conflicts of interest to this work. And they did not have a direct financial relation with the commercial identity mentioned in this paper.

\section{Acknowledgment}

This work is financially supported by the National Science and Technology Major Project (2009ZX10004-601, 2012ZX10005001-004, and 2012ZX10005001-008), Shanghai Interdisciplinary Cultivation Platform of Outstanding and Innovative Postgraduates.

\section{References}

[1] W. Jia, L. P. Zhao, and Z. Chen, "Systems biomedicine-the convergence of western medicine and traditional Chinese medicine," World Science and Technology/Modernization of Traditional Chinese Medicine and Materia Medica, vol. 9, no. 2, pp. 1-5, 2007.

[2] X. Wu, R. Jiang, M. Q. Zhang, and S. Li, "Network-based global inference of human disease genes," Molecular Systems Biology, vol. 4, no. 189, pp. 1-11, 2008.

[3] J. Warner, "Blood pressure drug may help treat MS-study shows lisinopril blocks multiple sclerosis in lab mice," Web MD Health News, 2009.

[4] M. I. Fonseca, R. R. Ager, S. H. Chu et al., "Treatment with a $\mathrm{C} 5 \mathrm{aR}$ antagonist decreases pathology and enhances behavioral performance in murine models of Alzheimer's disease," Journal of Immunology, vol. 183, no. 2, pp. 1375-1383, 2009.

[5] J. B. Zhang, "Complete works of Jingyue (jing yue quan shu)," 1640.

[6] T. S. Ye, “Treatise on worm heat disease (wen bing lun)," 1746.

[7] World Health Organization, WHO International Standard Terminologies on Traditional Medicine in the Western Pacific Region, WHO Library Cataloguing in Publication Data, 2007.

[8] Z. Wang, J. Wang, and Y. Y. Wang, "Thinking of Omic study in TCM Syndrome at the post-genomic era," Chinese Journal of Integrated Traditional and Western Medicine, vol. 21, no. 8, pp. 621-623, 2001.

[9] Y. Y. Wang, Q. M. Zhang, and Z. B. Zhang, "The induction of syndrome factor and affecting target," Journal of Shandong University of TCM, vol. 30, no. 1, pp. 6-7, 2006.

[10] J. L. Tang, B. Y. Liu, and K. W. Ma, “Traditional Chinese medicine," The Lancet, vol. 372, no. 9654, pp. 1938-1940, 2008.

[11] H. Y. Wang, H. M. Li, B. Yang, and J. J. Xu, “The relationship between distribution characteristics of TCM constitution and syndromes in 141 patients with fatty liver," Journal of Beijing University of TCM, vol. 33, no. 7, pp. 500-502, 2010.

[12] J. Chang, X. F. Pan, W. W. Qiu, G. Y. Yang, and L. Q. Wang, "Objectivized study on syndrome differentiation for chronic hepatitis B," Jiangsu Journal of TCM, vol. 27, no. 5, pp. 26-27, 2006.

[13] "The guideline of prevention and treatment for chronic hepatitis B, (2010 version)," Chinese Journal of Hepatology, vol. 19, no. 1, pp. 13-24, 2011.

[14] "Guidelines for management of nonalcoholic fatty liver disease: an updated and revised edition," Chinese Journal of Hepatology, vol. 18, no. 3, pp. 163-166, 2010.
[15] S. J. Sun, J. Y. Dai, W. Y. Wang et al., "Metabonomic evaluation of ZHENG differentiation and treatment by fuzhenghuayu tablet in hepatitis-B-caused cirrhosis," Evidence-Based Complementary and Alternative Medicine, vol. 2012, Article ID 453503, 8 pages, 2012.

[16] L. Eriksson, E. Johansson, and N. Kettaneh-Wold, Multi and Megavariate Data Analysis, Part 1: Basic Principles and Applications, Umetrics AB, Umeå, Sweden, 2nd edition, 2001.

[17] P. Yin, D. Wan, C. Zhao et al., "A metabonomic study of hepatitis $\mathrm{B}$-induced liver cirrhosis and hepatocellular carcinoma by using RP-LC and HILIC coupled with mass spectrometry," Molecular BioSystems, vol. 5, no. 8, pp. 868-876, 2009.

[18] C. Heßlinger, S. A. Fairhurst, and G. Sawers, "Novel keto acid formate-lyase and propionate kinase enzymes are components of an anaerobic pathway in Escherichia coli that degrades Lthreonine to propionate," Molecular Microbiology, vol. 27, no. 2, pp. 477-492, 1998.

[19] C. Ingram-Smith, A. Gorrell, S. H. Lawrence, P. Iyer, K. Smith, and J. G. Ferry, "Characterization of the acetate binding pocket in the Methanosarcina thermophila acetate kinase," Journal of Bacteriology, vol. 187, no. 7, pp. 2386-2394, 2005.

[20] W. J. Qi, S. W. Zhang, and H. Wang, "Modern research progress of damp-heat syndrome," JETCM, vol. 19, no. 7, pp. 1200-1201, 2010.

[21] F. P. Cheng, S. L. Liu, Y. H. Li, X. Zhou, and G. Q. Mei, "The study of effects of Qingre Huashi Fang in febrile disease Dampness-heat Syndrome rat liver mitochondria of $\mathrm{Na}^{+}-\mathrm{K}^{+}$ATPase," Hubei Journal of TCM, vol. 29, no. 3, pp. 3-5, 2007.

[22] X. S. Huang, "The research of $\mathrm{Na}^{+}-\mathrm{K}^{+}$-ATPase activity in erythrocyte membrane in rats with Wet-resistance in middlejiao," Chengdu Journal of TCM, vol. 23, no. 4, pp. 23-24, 2004.

[23] C. Hari, I. Raphael, and R. Rajendram, "Compartmentalization of aquaporins in the human intestine," International Journal of Environmental Research and Public Health, vol. 5, no. 2, pp. 115119, 2008.

[24] S. J. Wu and L. B. Liao, "Changes of aquaporin AQP2 content in rats' renal medullary and urine with Dampness-heat syndrome," Chinese Medicine Technology, vol. 10, no. 1, pp. 4-5, 2003.

[25] X. Yan and M. Y. Guo, "Observation on pathological changes of tongue in rats with febrile disease of dampness-over-heat syndrome," Journal of Anhui TCM College, vol. 24, no. 1, pp. 1921, 2005.

[26] J. F. Martín, "Phosphate control of the biosynthesis of antibiotics and other secondary metabolites is mediated by the PhoR-PhoP system: an unfinished story," Journal of Bacteriology, vol. 186, no. 16, pp. 5197-5201, 2004.

[27] M. A. Kertesz, A. M. Cook, and T. Leisinger, "Microbial metabolism of sulfur-and phosphorus-containing xenobiotics," FEMS Microbiology Reviews, vol. 15, no. 2-3, pp. 195-215, 1994.

[28] R. K. Jain, M. Kapur, S. Labana et al., "Microbial diversity: application of microorganisms for the biodegradation of xenobiotics," Current Science, vol. 89, no. 1, pp. 101-112, 2005.

[29] I. Elmadfa, D. Majchrzak, P. Rust, and D. Genser, “The thiamine status of adult humans depends on carbohydrate intake," International Journal for Vitamin and Nutrition Research, vol. 71, no. 4, pp. 217-221, 2001.

[30] World Health Organization, "Thiamine deficiency and its prevention and control in major emergencies," Report WHO/NHD/99.13, Department of Nutrition for Health and Development, WHO, Geneva, Switzerland, 1999. 
[31] A. Fattal-Valegski, A. Kesler, B. Sela et al., "Outbreak of life threatening thiamine deficiency in infants in Israel caused by a defective soy based formula," Pediatrics, vol. 115, pp. 33-38, 2005.

[32] Z. B. Wu, S. Q. Peng, P. Z. Lin, J. X. Zhong, and Q. Shen, "Effects of damp-heat environment on lipopolysaccharide induced bactericidal/permeability-increasing protein mRNA expression," Journal of Traditional Chinese Medicine, vol. 7, no. 9, pp. 40-42, 2001. 


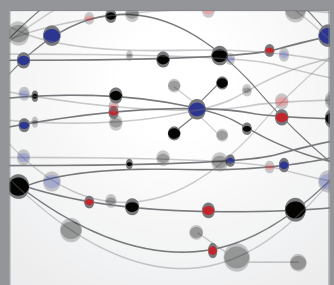

The Scientific World Journal


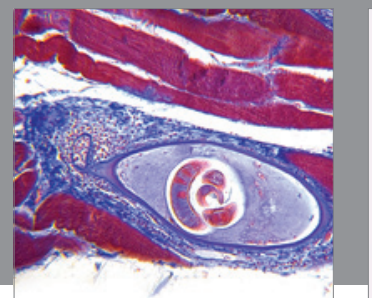

Gastroenterology

Research and Practice
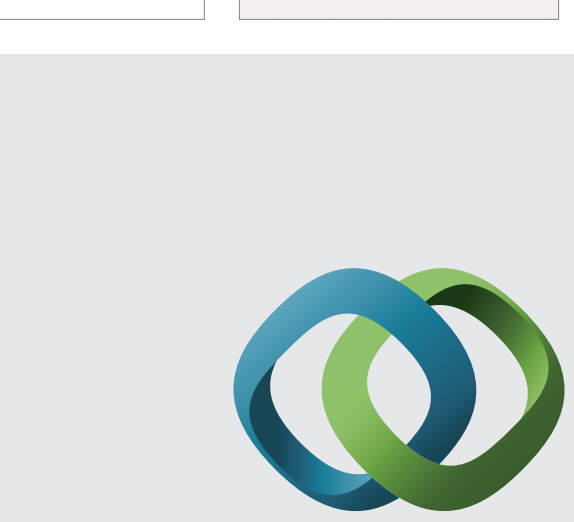

\section{Hindawi}

Submit your manuscripts at

http://www.hindawi.com



Disease Markers
